\title{
Appendix Neuroendocrine Tumor pT4 TNM Finding v8
}

National Cancer Institute

\section{Source}

National Cancer Institute. Appendix Neuroendocrine Tumor pT 4 TNM Finding v8. NCI

Thesaurus. Code C135150.

Appendix neuroendocrine tumor perforating the peritoneum or directly invading other adjacent organs or structures (excluding direct mural extension to adjacent subserosa of adjacent bowel), e.g., abdominal wall and skeletal muscle. (from AJCC 8th Ed.) 